\title{
Reliability and robustness of rainfall compound distribution model based on weather pattern sub-sampling
}

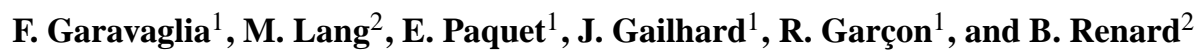 \\ ${ }^{1}$ EDF - DTG, 21 Avenue de l'Europe, BP 41, 38040 Grenoble Cedex 9, France \\ ${ }^{2}$ CEMAGREF, UR HHLY, Hydrology-Hydraulics, 3bis quai Chauveau, CP220, 69366 Lyon Cedex 09, France
}

Received: 30 July 2010 - Published in Hydrol. Earth Syst. Sci. Discuss.: 7 September 2010

Revised: 28 December 2010 - Accepted: 3 February 2011 - Published: 9 February 2011

\begin{abstract}
A new probabilistic model for daily rainfall, named MEWP (Multi Exponential Weather Pattern) distribution, has been introduced in Garavaglia et al. (2010). This model provides estimates of extreme rainfall quantiles using a mixture of exponential distributions. Each exponential distribution applies to a specific sub-sample of rainfall observations, corresponding to one of eight typical atmospheric circulation patterns that are relevant for France and the surrounding area.

The aim of this paper is to validate the MEWP model by assessing its reliability and robustness with rainfall data from France, Spain and Switzerland. Data include 37 long series for the period 1904-2003, and a regional data set of 478 rain gauges for the period 1954-2005. Two complementary properties are investigated: (i) the reliability of estimates, i.e. the agreement between the estimated probabilities of exceedance and the actual exceedances observed on the dataset; (ii) the robustness of extreme quantiles and associated confidence intervals, assessed using various sub-samples of the long data series. New specific criteria are proposed to quantify reliability and robustness. The MEWP model is compared to standard models (seasonalised Generalised Extreme Value and Generalised Pareto distributions). In order to evaluate the suitability of the exponential model used for each weather pattern (WP), a general case of the MEWP distribution, using Generalized Pareto distributions for each WP, is also considered.

Concerning the considered dataset, the exponential hypothesis of asymptotic behaviour of each seasonal and weather pattern rainfall records, appears to be reasonable. The results highlight : (i) the interest of WP sub-sampling
\end{abstract}

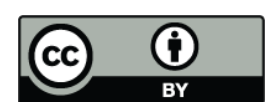

Correspondence to: F. Garavaglia (federico-externe.garavaglia@edf.fr) that lead to significant improvement in reliability models performances; (ii) the low level of robustness of the models based on at-site estimation of shape parameter; (iii) the MEWP distribution proved to be robust and reliable, demonstrating the interest of the proposed approach.

\section{Introduction}

The distributions of hydrologic variables such as rainfall and streamflow play a key role in the design of water-related infrastructures (i.e. dam spillways or river dikes). The objective of hydrologic design is to quantify and mitigate the flood risk arising from high rainfall and streamflow values. The methods used for the computation of flood risk for extreme floods can be devised into two families: the deterministic methods and the probabilistic methods. The deterministic models approach this issue from a physic point of view and they are based on the concept of Probable Maximum Flood (PMF). The PMF can be defined as the flood that may be expected from the most severe combination of critical meteorological and hydrologic conditions that are reasonably possible in a particular drainage area. On the other hand the probabilistic methods based on statistic models treat the problems in terms of probability (or equivalently in terms of return level) introducing the concept of flood distribution.

Historically in French context the probabilistic method are preferred to the deterministic ones. More precisely EDF design floods for dam spillway have been computed using the Gradex method since 1970 (Guillot and Duband, 1967; CFGB, 1994). This method is based on the assumptions that: (i) extreme rainfalls are realizations from an exponential distribution, and (ii) when the catchment is close to saturation, each increase of rainfall $d P$ induces an equivalent

Published by Copernicus Publications on behalf of the European Geosciences Union. 
increase of discharge $d Q$. This implies an asymptotic parallelism between rainfall and discharge cumulative distribution functions (cdf) plotted in Gumbel axes. The Gradex method therefore extrapolates the flood distribution beyond a return period $T_{\mathrm{g}}$, using the scale parameter (called the gradex parameter) of the rainfall distribution. Assumptions (i) and (ii) may appear too restrictive, as the former underestimates the rainfall distribution with an excessive number of exceedances of 10-year rainfall quantiles (Garçon, 1995), and the latter overestimates the rate of the discharge cdf near the return period $T_{\mathrm{g}}$ (asymptotic parallelism considered to be effective from $T_{\mathrm{g}}$ ). So far, EDF has a positive feedback: there is no significant indication of under-estimation of design flood on a dataset of 450 hydrologic designs. But there was a need to assess both the rainfall and discharge hazards in more depth. This is one of the reasons that have promoted the development of the Schadex method (Paquet et al., 2006). SCHADEX uses a semi-continuous simulation process for flood frequency estimation. This process is based on historical observed rainfall and temperature time series. Major observed rainfall events are replaced by randomly drawn synthetic events, whose probability is issued from the MEWP (Multi-Exponential Weather Pattern) distribution. The MEWP distribution, is a mixture of exponential distributions fitted on rainfall sub-samples based on a weather pattern classification (Garavaglia et al., 2010). These synthetic events are used as input of a rainfall-runoff model, which produces simulated streamflow events. This stochastic simulation is looped numerous times to combine almost exhaustively precipitation and hydrological risks.

The aims of this paper are to validate the MEWP distribution and to compare it with standard probabilistic models stemming from extreme value theory. To this aim, specific criteria quantifying the models performance in terms of reliability and robustness are proposed. This assessment is based on a large dataset of daily rainfall series located in France, Switzerland and Spain. The paper is organized as follows: Sect. 2 summarizes the standard sampling techniques used in hydrological applications and details the probabilistic models used in this paper. The rainfall data set is presented in Sect. 3, and 4 describes the criteria used to evaluate the reliability and robustness of the different probabilistic models. Results of the comparison are presented in Sect. 5, before drawing some conclusions and discussing potential improvements in Sect. 6.

\section{Sampling techniques and probabilistic models for extreme values}

This section describes the standard sampling techniques used in extreme value analysis and two additional sampling techniques (seasonal and weather pattern sub-sampling) commonly used in hydrological applications. It also describes the probabilistic models, the method used to estimate model parameters, and the computation of confidence intervals.

\subsection{Standard sampling techniques}

Two standard sampling techniques are used to build samples of extreme values:

- Block Maximum (BM). The maximum values within blocks of equal length are selected. The choice of block size is important as too small blocks can lead to bias and too large blocks generate too few block maxima, thus yielding a large estimation variance (Coles, 2001). Usually a one-year block is used for daily discharges or rainfall data, yielding annual maxima (AM) series. Asymptotic considerations suggest that the distribution of AM can be approximated by a generalized extreme value (GEV) distribution (Coles et al., 2003).

- Peaks over threshold (POT). All events exceeding a given threshold are selected (see Lang et al., 1999; Rosbjerg and Madsen, 2004, for a review). According to Coles (2001), such a sample may be considered as independent realizations of a random variable whose distribution can asymptotically (i.e., for high thresholds) be approximated by a generalized Pareto (GP) distribution.

According to Coles et al. (2003), if daily series are available, POT sampling may be more efficient than AM sampling, because additional information on several large events occurring during the same year is taken into account.

\subsection{Seasonal and weather patterns sampling techniques}

Seasonal sampling is widely used in hydrological applications (Leonard et al., 2008) and overall considered as essential in precipitation analysis. This kind of stratification is often performed to produce more homogeneous sub-samples than the whole population (Lang et al., 1994; Djerboua and Lang, 2007). Several studies have shown that in the Mediterranean area of Europe (French, Spanish and Italian regions) extreme rainfall events are mainly observed between the end of summer and autumn (Zveryaev, 2004; Müller et al., 2009; Karagiannidis et al., 2009). Consequently, we will define a "Season-at-Risk" period as the three consecutive months with highest monthly rainfall maxima. All the presented study will be carried out on this "Season-at-Risk". The definition of this seasonal sampling will be presented in the following section.

A number of authors have shown (e.g. Bardossy et al., 1995; Trigo and DaCamara, 2000; Linderson, 2001) that within the same season, the rainfall hazard in a specific area strongly depends on the atmospheric situation. The relationship between large-scale atmospheric circulation and precipitation events has been extensively studied (see Yarnal, 2001; Boé and Terray, 2008; Martinez et al., 2008). It has 
Table 1. Cumulative distribution functions and related sampling method. Label $x$ is used for maxima sampling, $y$ for POT sampling, and $z$ for POT and WP sampling.

\begin{tabular}{lcc}
\hline & Distribution function & Sampling \\
\hline GUM & $F(x \mid \mu, \lambda)=\exp \left[-\exp \left\{-\left(\frac{x-\mu}{\lambda}\right)\right\}\right]$ & Seasonal Maxima \\
GEV & $F(x \mid \mu, \lambda, \xi)=1-\exp \left(-\left[1+\xi\left(\frac{x-\mu}{\lambda}\right)\right]^{-1 / \xi}\right)$ & \\
\hline EXP & $F(y \mid \lambda)=1-\exp \left(\frac{y}{\lambda}\right)$ & Seasonal POT \\
GPD & $F(y \mid \lambda, \xi)=1-\left(1+\xi \frac{y}{\lambda}\right)^{-1 / \xi}$ & \\
\hline MEWP & $F\left(z \mid \lambda_{1}, \ldots, 8\right)=\sum_{i=1}^{8}\left(1-\exp \left[-\frac{z}{\lambda_{i}}\right]\right) \cdot p_{i}$ & Seasonal and WP POT \\
MGPWP & $F\left(z \mid \lambda_{1}, \ldots, 8, \xi_{1, \ldots, 8}\right)=\sum_{i=1}^{8}\left(1-\left[1+\xi_{i} \frac{z}{\lambda_{i}}\right]\right.$ & \\
\hline
\end{tabular}

been demonstrated that the analysis of the synoptic situation can provide significant information on heavy rainfall events (Littmann, 2000). Consequently, the rainfall probabilistic model of the SCHADEX method (Paquet et al., 2006) is based on this type of clustering. A specific Weather Pattern (WP) classification was developed (Garavaglia et al., 2010). It classifies each day into one of eight contrasted synoptic situations for France and surrounding areas, without seasonal distinction.

\subsection{Probabilistic models}

Table 1 describes the six probabilistic models considered in this study. The MEWP distribution is a particular case of the Multi Generalized Pareto Weather Patterns (MGPWP) distribution. Both probabilistic models are introduced by Garavaglia et al. (2010). They are based on the same concept: the seasonal rainfall records are split into several sub-samples corresponding to each WP. For the MEWP, an exponential distribution is fitted on a POT sampling of each WP subsample. For the MGPWP, a GP distribution is used. The seasonal distribution is then defined as the composition, for a given season, of all WP sub-sample marginal distributions, weighted by the relative occurrence of each WP. A comprehensive discussion on the threshold selection can be found in Garavaglia et al. (2010). Those mixture distributions will be compared to four standard models: the Gumbel (GUM) and the GEV distributions for AM samples, and the Exponential (EXP) and the GP distributions for POT samples.

The parameters of the six probabilistic models are estimated using the maximum likelihood method. The compound models (MEWP and MGPWP distributions) have more parameters than the standard probabilistic models. The MEWP and the MGPWP distributions have respectively 8 (one scale parameter for each WP) and 16 fitted parameters (one scale and one shape parameter for each WP). One of the goals of the comparison carried out in this paper is to assess the potential over-parameterisation of these models.
Note that the weights $p_{i}$ (see Table 1), equal to the frequency of each WP within a given season are directly computed from the daily time series of WP. They may or not be considered as parameters of the MEWP and MGPWP models: our choice is not to call them parameters because they are computed rather than fitted. Anyway, as the number of parameters is not explicitly accounted in the computed criteria, this does not affect the presented results.

Confidence intervals are computed using the nonparametric bootstrap technique (Efron, 1979). Random sampling with replacement from the initial sample produces new Bootstrap samples with the same length as the initial sample. For all $B$ bootstrap samples, the p-quantile $q_{\mathrm{p}}$ is computed with each probabilistic model, yielding a sample of $B$ quantile estimates $\left(q_{\mathrm{p}}^{(i)}\right)_{i=1 \ldots B}$. The confidence interval at $(1-\alpha)$ level is then equal to $\left[q_{\mathrm{p}, \alpha / 2}, q_{\mathrm{p}, 1-\alpha / 2}\right]$, where $q_{\mathrm{p}, \alpha / 2}, q_{\mathrm{p}, 1-\alpha / 2}$ are the empirical quantiles at values $\alpha / 2$ and $1-\alpha / 2$ computed from $\left(q_{\mathrm{p}}^{(i)}\right)_{i=1 \ldots B}$.

\section{Precipitation and their preprocessing}

The validation of the rainfall mixture distribution model is based on an extensive dataset composed of two daily rainfall archives:

- Dense dataset: data from 1502 rain gauges belonging to EDF, the French meteorological office Météo-France, the Swiss meteorological office Météo-Swiss and the Spanish meteorological office Instituto Nacional de Meteorología (INM) for the period 1953-2005. These stations are located in the Alps, Pyrenees and Massif Central at an average altitude of $622 \mathrm{~m}$.

- Long dataset: 308 long series from Météo-France covering the period 1904-2003. These stations are mainly located in the plain at an average altitude of $305 \mathrm{~m}$. 
Table 2. Characteristics of the rainfall data sets.

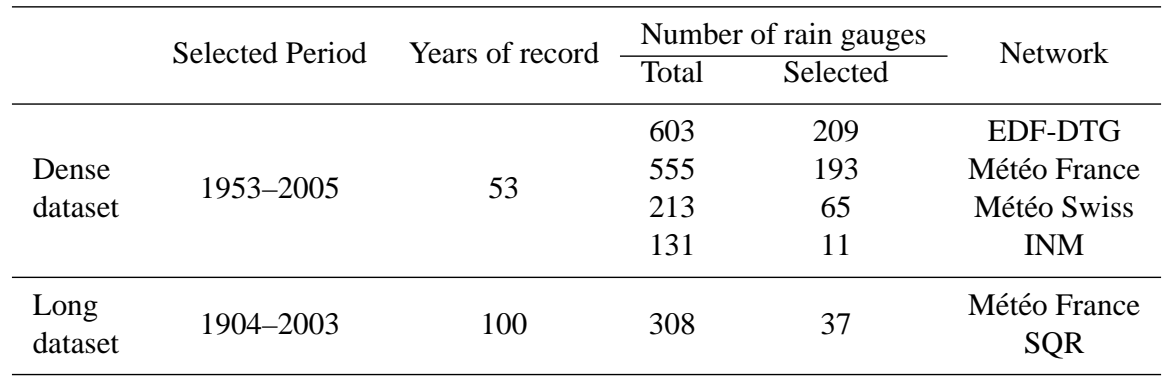
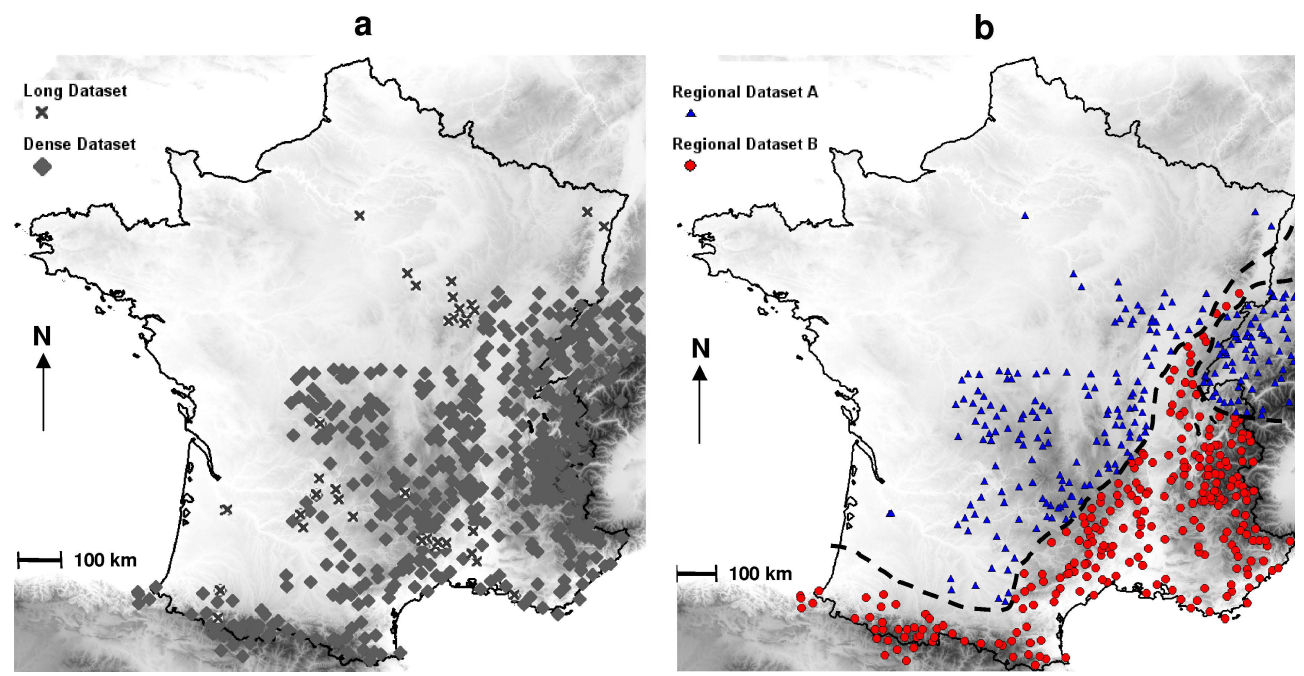

Fig. 1. (a) Rain gauges location. (b) Regional classification as a function of the "Season-at-risk", i.e. the three consecutive months that maximize the sum of the monthly rainfall maxima.

Both original datasets were first subject to a quality-check analysis, thus reducing the number of stations available for the model comparison. Only series with less than $10 \%$ of missing values per year were considered. Moreover, these series were further analysed to detect several anomalies: time shifts due to sensor replacement or station relocation, step changes or trends in rainfall intensity series.

The step change anomalies were studied by testing the stability over time of the residual of a multiple linear regression linking observations of the studied rain gauge with observations at the neighbouring rain gauges (Peterson and Easterling, 1994; Gottardi, 2009). Two statistics were combined in this test, based on the Alexandersson homogeneity test (Alexandersson, 1986) and of the sum of residuals with associated confidence intervals (Bois, 1976). Various tests are available for trend detection. In this study, we chose distribution-free tests because they do not require hypotheses on the data distribution (Hamed, 2009). According to Lang et al. (2006), two tests are commonly used to detect trends in non auto-correlated data series with unknown distribution: the Mann-Kendall test (Mann, 1945; Kendall, 1975) and Spearman's rho test (Lehmann, 1975; Sneyers, 1990).
The Mann-Kendall test was selected since it is as powerful as Spearman's rho test (Yue et al., 2002). 478 rain gauges from the dense dataset and 37 rain gauges from the long dataset were selected (Table 2) using this pre-processing. For both datasets, the most severe test has been the criterion on the percentage of missing value. For instance, concerning the long dataset, only 44 stations over 308 ( $14 \%$ ) were selected. Among these remaining series, the trends detection led to discard 7 more stations. Figure 1a shows the location of the selected stations from the two datasets.

For these datasets, the highest rainfalls occur at the end of the summer and during the autumn (from August to November). The "Season-at-risk" (Sect. 2.2) is computed for each rain gauge accordingly. The whole dataset (Long and Dense datasets) is divided into two datasets depending on the "Season-at-risk": the regional dataset A ("Seasonat-risk" from August to October) and the regional dataset B ("Season-at-risk" from September to November). Such a regional subdivision reveals a coherent spatial pattern, as shown in Fig. 1b. Figure 2a and $2 \mathrm{c}$ show the box plots of monthly rainfall maxima of regional datasets $\mathrm{A}$ and $\mathrm{B}$. As expected, the highest quantiles are reached between August 

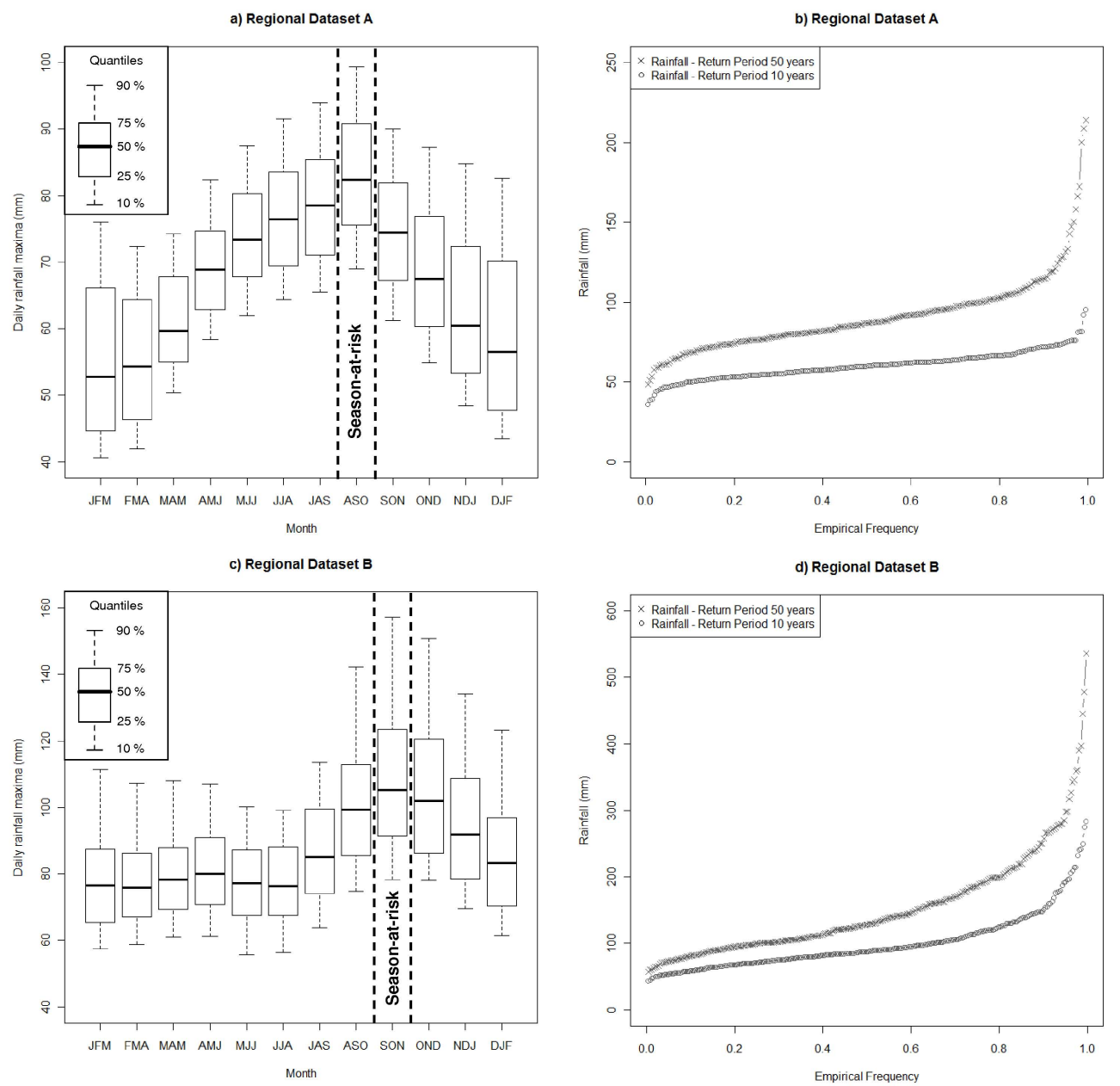

Fig. 2. Box plot of the three consecutive monthly rainfall maxima of regional dataset A (a) and regional dataset B (c). Empirical distribution of rainfall quantile estimates associated with 10- and 50-year return periods for regional dataset A (b) and regional dataset B (d).

and October (regional dataset A) or between September and November (regional dataset $B$ ). Figure $2 b$ and $d$ show that the two regional data sets cover a large variability of rainfall intensities, from 40 to $170 \mathrm{~mm}$ (resp. 40 to $290 \mathrm{~mm}$ ) for the empirical daily 10-year rainfall for dataset A (resp. B) and from 50 to $220 \mathrm{~mm}$ (resp. 70 to $520 \mathrm{~mm}$ ) for the empirical daily 50-year rainfall for the dataset A (resp. B).

\section{Comparison of probabilistic models}

This section describes the strategy used to compare the probabilistic models, and defines several criteria to quantify the reliability and robustness of each model. Several statistical tests are reported in the literature to measure the goodness of fit: Pearson's chi-square test (Plackett, 1983), Kolmogorov - Smirnov test (Kolmogorov, 1941; Smirnov, 1944), Anderson - Darling Test (Anderson and Darling, 1952), Cramervon-Mises criterion (Cramer, 1928; Darling, 1957), ShapiroWilk test (Shapiro and Wilk, 1965) and test of Lilliefors
(Lilliefors, 1967). These standard tests are not perfectly suited for extreme value distributions, mainly because they are not enough sensitive to deviations in the tails of the distribution. In order to take into account these limitations, several transformations of standard tests have been proposed (e.g. Khamis, 1997; Liao and Shimokawa, 1999; Laio, 2004). Applications of the Akaike information criterion (AIC) (Akaike, 1974) and on the Bayesian information criterion (BIC) (Schwarz, 1978) are also often found in the literature (e.g. Nacházel, 1993; Di Baldassarre et al., 2009; Laio et al., 2009). In contrast with the list of standard tests given above, the AIC and BIC criteria introduce a penalty term for the number of parameters. Laio et al. (2009) evaluated their capability to identify the correct parent distribution from the available data and showed that these criteria perform well if the parent distribution is a two-parameter distribution. In contrast, they are less efficient in the case of three-parameters distribution. 
This paper does not solely focus on goodness of fit, and instead attempts to evaluate the predictive performance of a model using independent validation data (i.e. not used to calibrate the model). Moreover, focus is on the tail of the distribution, i.e. the performance of the model in estimating the exceedance probability of large values. It is argued that the evaluation of goodness-of-fit is not sufficient to assess the ability of a model to predict the exceedance probability of future (unobserved) values. Consequently, we propose an alternative approach based on specific criteria computed on an extensive dataset.

A probabilistic model of extreme rainfall should be both reliable and robust. A reliable model assigns the "correct" exceedance probability to high values. In practice, this property can only be evaluated with respect to observed data. Consequently, it is useful to consider both long series and dense data sets in order to increase the sample of observed extreme values. On the other hand, a robust probabilistic model yields similar estimates when a slight perturbation of data is introduced. This property is very important, especially in the extrapolation of extreme quantiles, in order to avoid an estimate being overly sensitive to sampling variability. Robustness is easier to quantify than reliability but an analysis solely based on the former is not sufficient because robustness does not give any information about the ability of the model to describe or predict observations. In the absence of reliability diagnostics, a robust model is not necessarily preferable: a model can be robust but totally unreliable. In conclusion these properties are complementary: the reliability of the model should be evaluated first, and in a second step, the most robust model (amongst reliable ones) should be preferred. Specific criteria quantifying reliability and robustness are proposed in the following sections.

\subsection{Reliability criteria}

As mentioned above, measuring the reliability of probabilistic estimations of high quantiles is not an easy task. We take cues from methods developed in the context of skill assessment of probabilistic forecasts, in particular, the reliability diagram (also called attribute diagram) (Wilks, 1995). This tool is used to assess the consistency of a probabilistic forecast of binary events. It plots the observed frequency against the forecast probability in order to evaluate their agreement. This diagram is widely used in forecasts analysis and comparisons (e.g. see Bartholmes et al., 2009, for an application).

Similarly, we propose a specific procedure to evaluate the agreement between the exceedance probabilities of extreme events provided by a probabilistic model and their observed frequencies. This tool, named $F F$ criterion, is based on a split-sample procedure and was introduced by Garçon (1995). Let $D$ be a regional data set of $L$ stations of length $N, D^{i}$ is the time series at site $i$. The computation of the $F F$ criterion can be divided into the following steps:
1. Each $D^{i}$ is split into two successive sub-samples of equal length $N / 2:\left(x_{1}^{i}, \ldots, x_{\mathrm{N} / 2}^{i}\right)$ and $\left(x_{\mathrm{N} / 2+1}^{i}, \ldots, x_{\mathrm{N}}^{i}\right)$.

2. Two cdf $F_{1}^{i}(x)$ and $F_{2}^{i}(x)$ of the same probabilistic model are fitted using each sub-sample.

3. Let $m_{1}^{i}=\max \left\{x_{1}^{i}, \ldots, x_{\mathrm{N} / 2}^{i}\right\} \quad$ and $\quad m_{2}^{i}=$ $\max \left\{x_{\mathrm{N} / 2+1}^{i}, \ldots, x_{\mathrm{N}}^{i}\right\}$. Under the hypothesis of i.i.d. random variables the probability of non-exceedance of $m_{1}^{i}$ (resp. $m_{2}^{i}$ ) is computed with the cdf fitted to the second part $F_{2}^{i}(x)$ (resp. the first part $F_{1}^{i}(x)$ ) as follows:

$$
\begin{aligned}
& F F_{1}^{i}=\operatorname{Pr}\left(M_{i} \leq m_{1}^{i}\right)=\left[F_{2}^{i}\left(m_{1}^{i}\right)\right]^{\mathrm{N} / 2} \\
& F F_{2}^{i}=\operatorname{Pr}\left(M_{i} \leq m_{2}^{i}\right)=\left[F_{1}^{i}\left(m_{2}^{i}\right)\right]^{\mathrm{N} / 2}
\end{aligned}
$$

$2 L$ values of probabilities $F F$ are therefore computed. With a perfect probabilistic model, the distribution of $F F$ values should be a Kumaraswamy's double bounded distribution of parameters $N$ and 1; i.e. $K[N, 1]$ (Kumaraswamy, 1980); see Appendix A. A pp-plot is used to check this feature: the closer the $F F$ distribution to the 1:1 diagonal, the more reliable the probabilistic model.

In practice, the theoretical distributions $F_{1}^{i}(x)$ and $F_{2}^{i}(x)$ are replaced by their estimates based on samples of limited size, thus leading to departures from the 1:1 line. To quantify this, $F F$ is calculated on 1000 random datasets of three different sample sizes, generated from an exponential population. The size of the first sample is similar to that of the actual rainfall dataset $(L=552, N=50)$, the second is smaller $(L=552, N=10)$ and the third is bigger $(L=552$, $N=1000$ ). Figure 3 shows the median of the simulated $F F$ distributions for each dataset size. It appears that logically, the $F F$ distribution plot moves closer to the 1:1 diagonal (theoretical result) when the sample size increases. Because of the bias introduced by the limited sample size, the analysis of the reliability test is mainly qualitative and provides a way to compare concurrent probabilistic models.

The $F F$ procedure is used to assess the ability of a probabilistic model to assign the "correct" probability to the highest observed values that were not used for model fitting. With analogy with the split sample test, this kind of procedure can be named $F F$ validation procedure. Note that the $F F$ procedure solely focuses on the maximum observed value during the validation period: it is therefore primarily geared toward the assessment of reliability in the tail of the distribution.

A modification of the $F F$ validation procedure can be introduced in order to assess reliability based on the calibration sub-sample. Instead of computing the non-exceedance probability of the maximum of the first sub-sample with the cdf estimated on the second sub-sample, the cdf fitted on the 


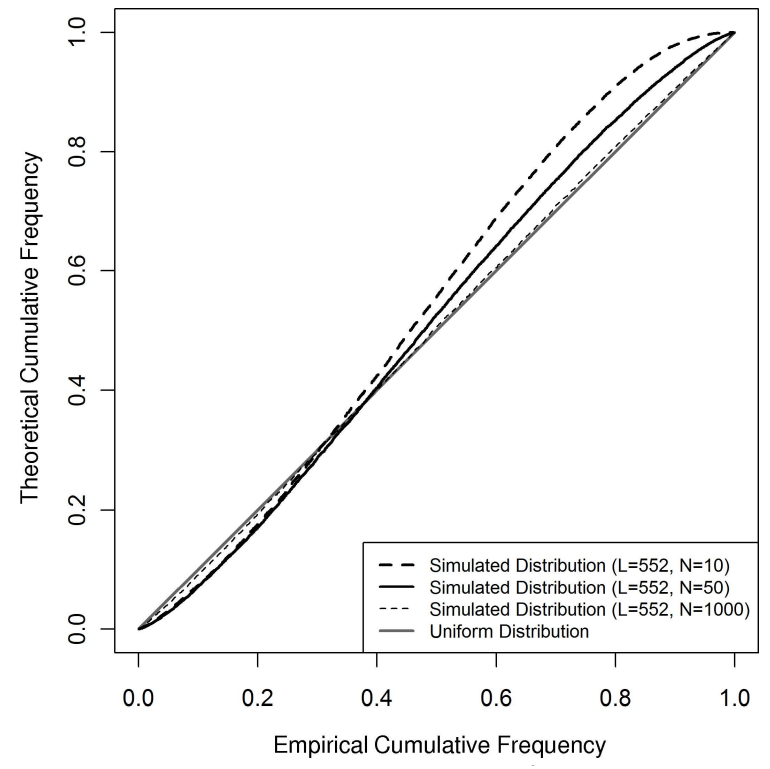

Fig. 3. $F F$ distribution provided by simulation with random samples extracted from an exponential distribution. Different curves represent three kinds of simulations with samples of different sizes.

same sub-sample can be used:

$$
\begin{aligned}
& \left(F F_{1}^{i}\right)^{*}=\operatorname{Pr}\left(M_{i} \leq m_{1}^{i}\right)=\left[F_{1}^{i}\left(m_{1}^{i}\right)\right]^{\mathrm{N} / 2} \\
& \left(F F_{2}^{i}\right)^{*}=\operatorname{Pr}\left(M_{i} \leq m_{2}^{i}\right)=\left[F_{2}^{i}\left(m_{2}^{i}\right)\right]^{\mathrm{N} / 2}
\end{aligned}
$$

This approach can be interesting in cases where the observed distribution of $F F^{*}$ values is less variable than the theoretical $K[N, 1]$ distribution. Indeed, the latter distribution corresponds to what should be observed using the true distribution of data: it corresponds to a lower bound for the variability of $F F^{*}$ values, solely resulting from sampling variability. Consequently, a probabilistic model yielding $F F^{*}$ values less variable than the theoretical $K[N, 1]$ distribution tends to "over-fit" extreme values, which is typical of overparameterized models. With analogy to the $F F$ validation procedure, this second approach can be named the $F F$ calibration procedure.

In order to improve the comparison a robustness assessment is presented into the following paragraph.

\subsection{Robustness criteria}

The robustness is the ability of a method to yield close estimations when two different calibration periods are utilised. Robustness is quantified using several sub-samples of the whole long data series, in order to increase the reliability of the assessment. To analyse the results and compare the models, two scores are computed: the $\operatorname{SPAN}_{\mathrm{T}}$ criterion and the COVER $_{\mathrm{T}}$ criterion.
The SPAN $_{T}$ criterion aims to evaluate the variability of extreme quantile estimation. This criterion can be defined as follows:

$$
\operatorname{SPAN}_{\mathrm{T}}=\frac{\max \left\{\hat{q}_{T, n=1, \ldots, m}\right\}-\min \left\{\hat{q}_{T, n=1, \ldots, m}\right\}}{\frac{1}{m} \sum_{\mathrm{n}=1}^{m} \hat{q}_{\mathrm{T}, \mathrm{n}}}
$$

where $\hat{q}_{\mathrm{T}, \mathrm{n}}$ is the model estimate for the return period $T$ and the sub-period $n$ amongst $m$ non-overlapping sub-periods. The value of this score is greater or equal to 0 , zero being the ideal score, occurring for a probabilistic model that is completely unaffected by the sub-period used for calibration.

Moreover, it is reasonable to assert that a probabilistic model is more robust if the confidence intervals calculated for different sub-periods overlap well. Note that we are interested here in confidence interval overlap and not in their width. Indeed, for a given model and return period, two bootstrap confidence intervals (computed from two different sub-samples) could be narrow but totally disconnected. Such behavior is not in line with the robustness requirement. To quantify this property, a second criterion, named $\mathrm{COVER}_{\mathrm{T}}$ is derived. The analytical expression of this score is as follows:

\section{COVER $_{\mathrm{T}}$}

$=\frac{\prod_{\mathrm{n}=1}^{\mathrm{m}} \operatorname{Pr}\left(\max \left\{\hat{q}_{T, \alpha / 2, \mathrm{n}=1, \ldots, m}\right\} \leq \hat{q}_{\mathrm{T}, \mathrm{n}} \leq \min \left\{\hat{q}_{T, 1-\alpha / 2, \mathrm{n}=1, \ldots, m}\right\}\right)}{(1-\alpha)^{\mathrm{m}}}$

$=\frac{\prod_{n=1}^{\mathrm{m}} \operatorname{Pr}\left(a \leq \hat{q}_{\mathrm{T}, \mathrm{n}} \leq b\right)}{(1-\alpha)^{\mathrm{m}}}$

where $\hat{q}_{\mathrm{T}, \alpha, \mathrm{n}}$ is the model estimate for the return level $T$ with a confidence level $\alpha$ and computed on the sub-period $n$ (amongst $m$ non overlapping sub-periods). This is the normalized product of the probability densities of the $\hat{q}_{\mathrm{T}}$ quantile within the $a-b$ interval, where $a$ is the highest value of the lower limit of the confidence intervals and $b$ is the lowest value of the upper limit of the confidence intervals. This score therefore provides a quantitative value of the confidence interval overlap for each sub-period. The graphical explanation of the COVER $_{\mathrm{T}}$ criterion is shown in Fig. 4 for two sub-periods. This figure highlights that the optimum of the criterion is 1 (confidence intervals are identical), and the minimum value is 0 (confidence intervals are disconnected).

\subsection{Comparaison methodology}

In this paragraph the comparison methodology (in terms of reliability and robustness) is detailed. The datasets are divided into 25-years sub-periods: two sub-periods of 25 years for the dense dataset and four sub-periods of 25 years for the long dataset. The division diagram is shown in Fig. 5. According to the same division scheme (Fig. 5) the $F F$ validation and calibration procedures are computed using the different probabilistic models. In regards to the long dataset we have considered the couples $1^{\text {st }}-2^{\text {nd }}$ period (1904-1928 and $1929-1953)$ and $3^{\text {th }}-4^{\text {th }}$ period (1954-1978 and 1979-2003). Furthermore in order to quantify the robustness, the probabilistic models were calibrated on all sub-periods $(N=25$ 

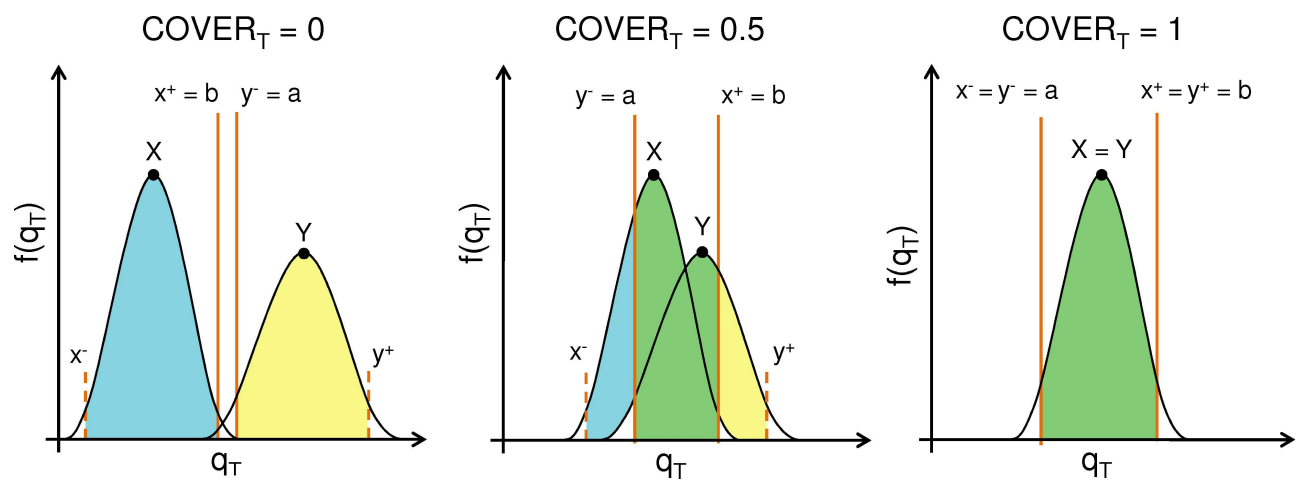

Fig. 4. Schematic confidence intervals overlap criteria: COVER $_{\mathrm{T}} . X$ is the model estimate computed on the sub-period 1 with confidence interval $\left[x^{-} ; x^{+}\right]$and $Y$ is the model estimate computed on the sub-period 2 with confidence interval $\left[y^{-} ; y^{+}\right] . a$ is the highest value of the lower limit of confidence intervals and $b$ is the lowest value of the upper limit of the confidence intervals. Three cases are shown: COVER $\mathrm{T}$ equal to 0 (null overlap), 0.5 (half overlap) and 1 (total overlap).

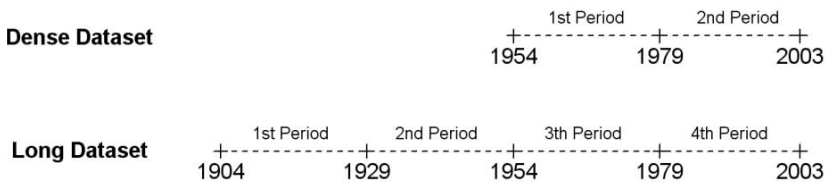

Fig. 5. Sub-period division of the two datasets.

years; $L=478+2 \cdot 37=552$ stations). SPAN $\mathrm{T}_{\mathrm{T}}$ and $\mathrm{COVER}_{\mathrm{T}}$ criteria are computed for each station and for different return levels.

Alternative division schemes, yielding sub-periods with different length and/or random sub-periods (i.e. containing non-consecutive years) were also tested in order to check that the results were not influenced by climatic effects or by the relative length of calibration/validation periods. The division scheme presented in Fig. 5 and these alternative division schemes led to similar results, so for a practical reason the latter results are not presented.

\section{Results}

This section presents the results of the model comparison. The GUM (resp. GEV) distribution performs closely to the EXP distribution (resp. GP) so for clarity's sake the scores of GUM and GEV distributions appear only in the tables and not in the figures of this section.

\subsection{Reliability}

Starting with the reliability criteria, the $F F$ calibration and validation criteria are calculated for the six models using the whole dataset. The results of these tests are presented through the pp-plot between the empirical and theoretical frequencies of the $F F$ values (Fig. 6). According to these results the MGPWP performs as well as MEWP distribution in validation but is the worst model in calibration. In particular, the shape of the MGPWP pp-plot in calibration suggests that the observed $F F$ values are less variable than theoretically expected. As indicated in Sect. 4.1, this is typical of over-parameterised models. Fitting the shape parameter on each WP sub-sample, the MGPWP distribution tends to over-fit extreme values. However, and perhaps surprisingly, this does not result in a loss of predictive performance in validation. Overall, and based on both criteria ( $F F$ calibration and validation criteria) the MEWP distribution is the most reliable model given that its distribution is the closest to the 1:1 diagonal.

Compared to MEWP and MGPWP distributions, the EXP and GP distributions have a distinctly lower predictive performance in validation (Fig. 6, right panel): this highlights the value of weather-pattern sub-sampling in estimating extreme quantiles. Moreover, the EXP distribution performs better than the GP distribution, which may appear surprising. Nevertheless this result is due to high variability of estimated shape parameter $\xi$ for the GP distribution. This parameter is sometimes negative, corresponding to an upper-bounded distribution. In such case, the $F F$ validation criterion is equal to 1 if the maximum observed value in the validation period is greater than the upper bound (corresponding to an "impossible" observation according to the model). In the whole dataset and in all sub-periods (1104 stations · periods) 632 negative shape parameter were estimated $(\sim 57 \%)$, yielding $\sim 9 \%$ of $F F$ values equal to 1 . These results highlight the limits of fitting the shape parameter using a few years of atsite data. On the contrary in the case of MGPWP distribution only $43(\sim 4 \%)$ negative shape parameter were estimated for the WP-at-risk (i.e. the WP associated to the highest scale parameter), yielding less then $\sim 1 \%$ of $F F$ values equal to one. These results show the interest of fitting the shape parameter on WP sample and not on the global population. This will be further discussed in Sect. 6. 

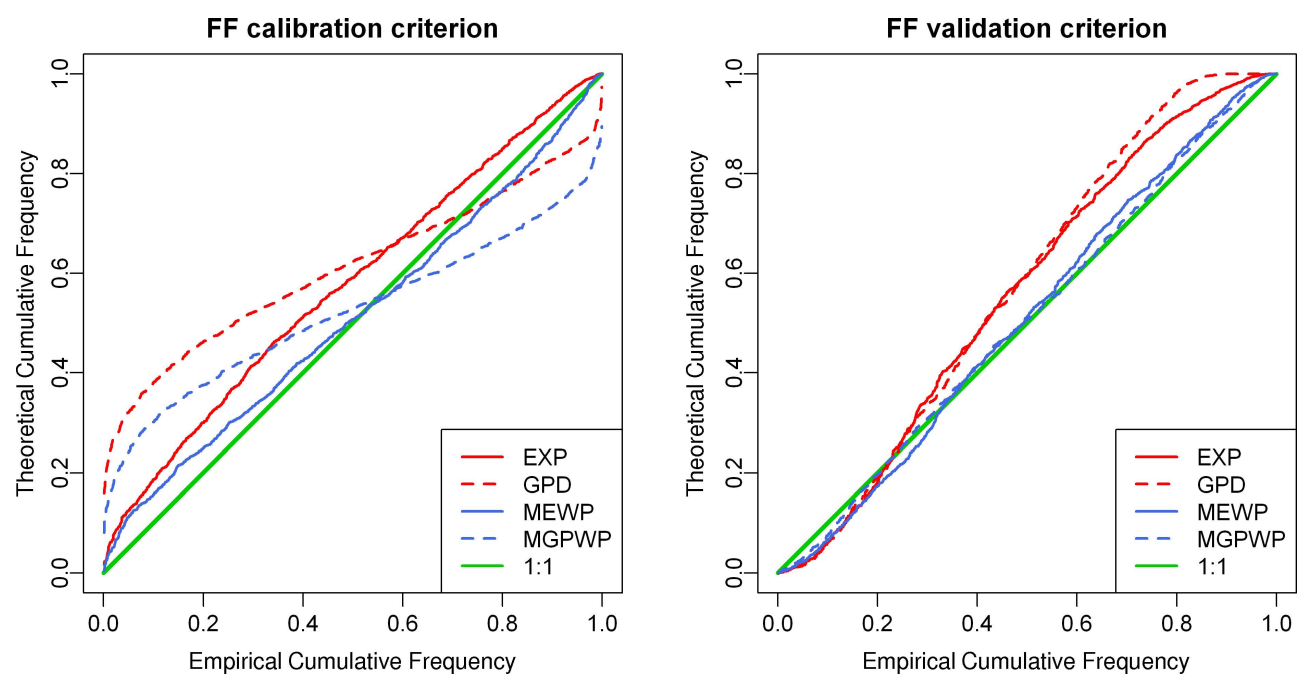

Fig. 6. pp-plot of $F F$ scores in calibration and validation. Whole dataset is used to computed these distributions.

Table 3. Results of the reliability procedure for the six probabilistic models.

\begin{tabular}{|c|c|c|c|c|}
\hline & & & $\frac{1}{1-f(F F)}$ & $\mathrm{f}(F F)$ \\
\hline \multirow{7}{*}{$\begin{array}{l}\text { A value } \\
\text { exceeded one } \\
\text { time over } 10 \\
\text { according to: }\end{array}$} & $\begin{array}{l}\text { Simulation EXP } \\
(M=552, N=50)\end{array}$ & \multirow{7}{*}{$\begin{array}{l}\text { is observed one } \\
\text { time over }\end{array}$} & 7 & 0.850 \\
\hline & GUM & & 5 & 0.784 \\
\hline & GEV & & 4 & 0.744 \\
\hline & EXP & & 5 & 0.780 \\
\hline & GPD & & 4 & 0.734 \\
\hline & MEWP & & 7 & 0.866 \\
\hline & MGPWP & & 8 & 0.869 \\
\hline \multirow{7}{*}{$\begin{array}{l}\text { A value } \\
\text { exceeded one } \\
\text { time over } 20 \\
\text { according to: }\end{array}$} & $\begin{array}{l}\text { Simulation EXP } \\
(M=552, N=50)\end{array}$ & \multirow{7}{*}{$\begin{array}{l}\text { is observed one } \\
\text { time over }\end{array}$} & 11 & 0.909 \\
\hline & GUM & & 7 & 0.860 \\
\hline & GEV & & 5 & 0.793 \\
\hline & EXP & & 7 & 0.864 \\
\hline & GPD & & 5 & 0.783 \\
\hline & MEWP & & 11 & 0.913 \\
\hline & MGPWP & & 15 & 0.931 \\
\hline \multirow{7}{*}{$\begin{array}{l}\text { A value } \\
\text { exceeded one } \\
\text { time over } 100 \\
\text { according to: }\end{array}$} & $\begin{array}{l}\text { Simulation EXP } \\
(M=552, N=50)\end{array}$ & \multirow{7}{*}{$\begin{array}{l}\text { is observed one } \\
\text { time over }\end{array}$} & 38 & 0.974 \\
\hline & GUM & & 16 & 0.938 \\
\hline & GEV & & 7 & 0.847 \\
\hline & EXP & & 17 & 0.941 \\
\hline & GPD & & 7 & 0.848 \\
\hline & MEWP & & 34 & 0.970 \\
\hline & MGPWP & & 32 & 0.969 \\
\hline
\end{tabular}

Particular attention has to be paid to the highest frequency in the presented pp-plot. In this regard, the $F F$ validation procedure may be expressed for high quantiles as follows. For example, with the EXP distribution the empirical cumulative frequency of the 0.95 quantile of $F F_{\mathrm{EXP}}$ is 0.86 (Fig. 7). This means that a value supposed to occur one time out of 20 , ac- cording to the EXP distribution $\left(F F_{\mathrm{EXP}}=0.95\right)$, has been observed about one time out of 7 (empirical cumulative frequency of 0.86). This kind of analysis has been done for each model (including the simulation using an exponential distribution presented in Sect. 4.1) and for different frequencies (0.9, 0.95 and 0.99). Table 3 illustrates the results of this 


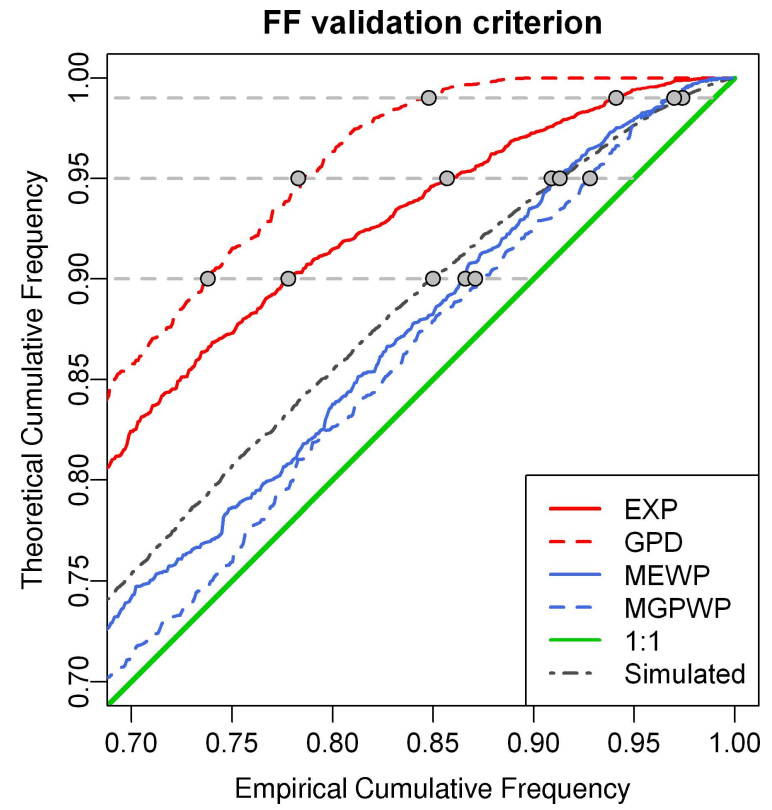

Fig. 7. Close-up of the upper tail of the $F F$ validation procedure. The gray circles highlight the values shown in Table 3. Whole dataset is used to computed these distributions.

analysis. It shows that the MEWP and MGPWP distributions are less biased than the other distributions, with observed values (resp. 7, 11 and 34 for the MEWP distribution and 8, 15, 32 for the MGPWP distribution) closer to both the theoretical values (resp. 10, 20 and 100) and the simulated values (resp. 7, 11 and 38) including the sampling effect (Fig. 3).

\subsection{Robustness}

Figure 8 shows the empirical distributions of the two robustness criteria $\left(\mathrm{SPAN}_{\mathrm{T}}\right.$ and $\mathrm{COVER}_{\mathrm{T}}$ ) computed at the 20-years, 100-years and 1000-years return levels. The GP and the MGPWP distributions are the most sensitive to sampling variability, as the SPAN 100 and SPAN $_{1000}$ scores are markedly larger than with the other distributions. The SPAN $_{20}$ remains almost similar for all the considered models (being MEWP the best one and MGPWP the worst one). Also in this case such a low level of robustness in these two models is due to high variations of the shape parameter $\xi$ in different sub-periods. Furthermore the MGPWP distribution drifts further away from the ideal $\mathrm{SPAN}_{\mathrm{T}}$ than GP distributions, especially for 1000-years return level. The other probabilistic models (EXP and MEWP distributions) yield similar and better SPAN $_{100}$ and SPAN 1000 scores.

In order to complete the robustness comparison, it is important to pay attention to the confidence interval overlap. The MEWP and the MGPWP distribution have the empirical distribution of the $\mathrm{COVER}_{20}$ score closest to the ideal score. Instead in the case of the empirical distribution of the COVER 100 and COVER 1000 scores, the MGPWP distri- bution performs better than the other ones. The good performance of MGPWP distribution in terms of $\mathrm{COVER}_{\mathrm{T}}$ criterion is a consequence of the width of its confidence intervals. Indeed, as the confidence intervals are wide, the probability to observe a good confidence interval overlap is higher. On the whole dataset, the MGPWP distribution at 100 -years return level has in average an interval confidence width equal to \pm 0.76 of the central estimation. The EXP, GP and MEWP distributions have respectively interval confidence width equal to $\pm 0.17, \pm 0.52$ and \pm 0.22 of the central estimation. The MEWP distribution yields satisfactory scores however its confidence interval size is appreciably moderate. The EXP and GP distributions are slightly less robust than the two distributions based on WP sub-sampling. Beside for these two models, the confidence intervals, computed on two different periods, appear totally disconnected for about $10 \%$ of the rain gauges (e.g. COVER $_{\mathrm{T}}$ score equal to 0 ).

A global robustness assessment may be summarized for the proposed criteria. Table 4 shows the mean SPAN $_{T}$ and COVER $_{\mathrm{T}}$ criteria at the 10-years, 20-years, 50-years, 100years and 1000-years return levels for the six probabilistic models considered. According to the results shown in Fig. 8 and in Table 4, the MEWP distribution provides a good level of robustness, from moderate to high return levels, either for the variability of extreme quantile estimation $\left(\mathrm{SPAN}_{\mathrm{T}}\right.$ criterion) or for confidence interval overlap $\left(\mathrm{COVER}_{\mathrm{T}}\right.$ criteria).

\section{Discussion and Conclusions}

The aim of this paper was to assess a probabilistic model based on atmospheric circulation pattern by comparing it with standard probabilistic models derived from extreme value theory using an extensive data set. A specific method for the comparison of probabilistic models was introduced. Firstly, the reliability of the model to estimate extreme rainfall quantiles was investigated. Secondly, the comparison examined the robustness of the extreme quantiles and their associated Bootstrap confidence intervals, based on various sub-samples of long data series (about 100 years). The use of long data series made it possible to compare the probabilistic models on extreme values. Seasonal variability of precipitation in France and in the surrounding area was taken into account.

Some interesting conclusions can be drawn. The results of the comparison clearly highlight the interest of a WP subsampling. In particularly the probabilistic models based on WP approach provide good predictive performance in validation ( $F F$ validation criterion). This conclusion means to suggest that the number of parameter, a priori a negative feature, does not affect the statistical qualities of the proposed probabilistic models based on WP.

For the GP and MGPWP distributions, the presented results shows that the shape parameter estimation leads to a 

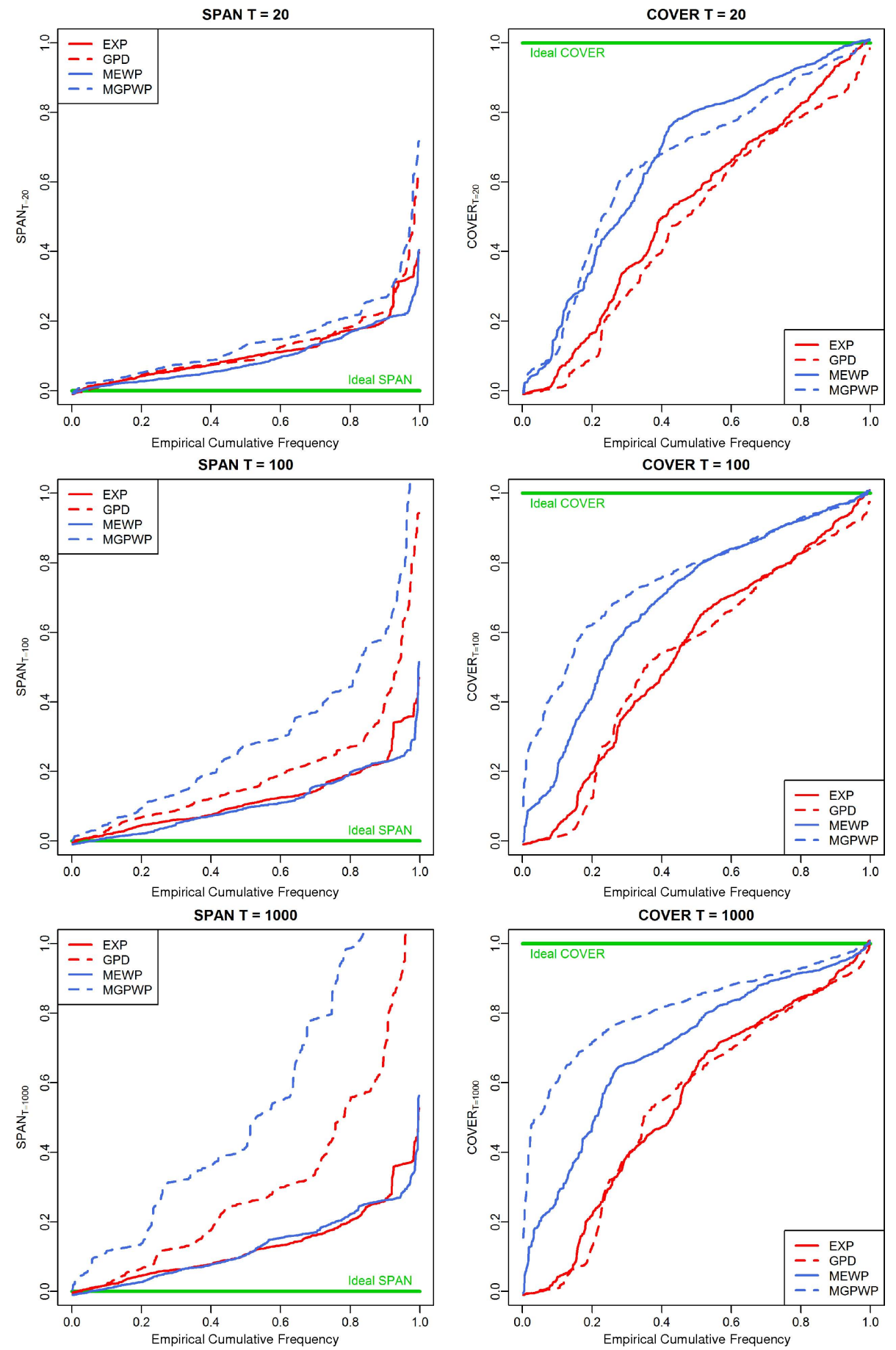

Fig. 8. Empirical distribution of $\mathrm{SPAN}_{\mathrm{T}}$ and $\mathrm{COVER}_{\mathrm{T}}$ criteria at 20 -years, 100 -years and 1000-years return levels. Whole dataset is used to computed these distributions.

drop in robustness, overall for high (100-years and 1000years) return levels. Therefore in operational application a regional analysis is recommended for robust estimation of shape parameter (Madsen et al., 1995; Martins et al., 2001; Ribatet et al., 2007; Pujol et al., 2008).
The purpose of this paper was to assess the MEWP probabilistic model and not to decry the GEV and GPD approach. As already said their observed low level of robustness is linked to the local estimation of the model parameters (especially the shape parameter $\xi$ ). Results for the MGPWP distribution are very contrasted. On the one hand a good level 
Table 4. Mean $\mathrm{SPAN}_{\mathrm{T}}$ and $\mathrm{COVER}_{\mathrm{T}}$ criteria (the numbers in bold highlight the best performance for each return period).

\begin{tabular}{crccccccc}
\hline Score & $\begin{array}{r}\text { Return period } \\
\text { (year) }\end{array}$ & Ideal score & GUM & GEV & EXP & GPD & MEWP & MGPWP \\
\hline \multirow{5}{*}{ SPAN $_{\mathrm{T}}$} & 10 & 0 & 0.09 & 0.10 & 0.09 & 0.10 & $\mathbf{0 . 0 8}$ & 0.10 \\
& 20 & 0 & 0.10 & 0.12 & 0.10 & 0.12 & $\mathbf{0 . 0 9}$ & 0.15 \\
& 50 & 0 & 0.11 & 0.16 & 0.12 & 0.16 & $\mathbf{0 . 1 0}$ & 0.22 \\
& 100 & 0 & $\mathbf{0 . 1 1}$ & 0.19 & 0.12 & 0.19 & $\mathbf{0 . 1 1}$ & 0.31 \\
& 1000 & 0 & $\mathbf{0 . 1 2}$ & 0.31 & 0.13 & 0.32 & 0.13 & 0.62 \\
\hline \multirow{3}{*}{ OVVER $_{\mathrm{T}}$} & 10 & 1 & 0.58 & 0.50 & 0.51 & 0.48 & $\mathbf{0 . 5 8}$ & 0.10 \\
& 20 & 1 & 0.59 & 0.53 & 0.53 & 0.48 & $\mathbf{0 . 6 7}$ & 0.66 \\
& 50 & 1 & 0.60 & 0.58 & 0.53 & 0.51 & 0.68 & $\mathbf{0 . 7 1}$ \\
& 100 & 1 & 0.60 & 0.60 & 0.54 & 0.53 & 0.69 & $\mathbf{0 . 7 6}$ \\
& 1000 & 1 & 0.61 & 0.64 & 0.55 & 0.54 & 0.70 & $\mathbf{0 . 8 1}$ \\
\hline
\end{tabular}

of $F F$ validation and $\mathrm{COVER}_{\mathrm{T}}$ criteria are observed, but on other hand this model presents a very low level of $F F$ calibration and SPAN $_{T}$ criteria. This aspect strongly reduces its applicability in operational application for reasons of coherence and repeatability. However we plan to carry out a future investigation on the use of a GP distribution for the most severe WP, with a regional assessment of the shape parameter.

In conclusion for daily data, the MEWP distribution presents a good level of reliability and robustness with respect to the proposed criteria. These conclusions may be different with sub-daily data. It would be interesting to carry out the same kind of study based on hourly time-series even if data availability would then be an issue especially for the robustness of the results.

In the proposed comparison technique the spatial dependence between samples maxima was not taken into account. The spatial dependence could influence the results of the $F F$ procedure, with a similar effect than the sampling effect presented in Fig. 3. However, the spatial dependence should not change the global results for a comparison purpose since all models are applied to the same data, affected by the same spatial dependence. Also we plan to carry out a future investigation on spatial distribution of computed scores and on correlation analyses between model performance and climatological features. The question of assessing the reliability (in addition to the robustness) of estimated uncertainties is also of interest. In our study the maximum likelihood method was used to fit models parameters. The uncertainties were not taken into account in the estimation of models parameters and so it could be potentially interesting to check if taking into account uncertainties (i.e. use a predictive distribution as models estimation, see Gelman et al., 1995) could improve reliability and robustness of models. Such developments are currently investigated within the French National research project named ExtraFlo 2009-2012 (EXTreme RAinfall and FLOod estimation: design values for extreme rainfall and floods. https://extraflo.cemagref.fr).

\section{Appendix A}

\section{Reliability criterion $\boldsymbol{F F}$}

Let:

- $D$ a regional dataset of $M$ stations;

- $D^{i}$ the time series at site $i$;

- $N^{i}$ the length of the $D^{i}$ time series;

- $m^{i}$ the observed maximum of $D^{i}$;

- $\hat{F}^{i}$ the probabilistic model fitted on $D^{i}$.

The $F F$ score at site $i$ can be defined as follow: $F F^{i}=\hat{F}^{i}\left(m^{i}\right)$

If the estimation is perfectly reliable $\left(\hat{F}^{i}=F^{i}\right)$, then $F F^{i} \sim$ $K\left[N^{i}, 1\right]$ (Kumaraswamy's double bounded distribution, Kumaraswamy, 1980), i.e. its cdf is $\operatorname{Pr}\left(F F^{i} \leq t\right)=t^{N^{i}}$ where $0 \leq t \leq 1$.

Proof:

$$
\begin{aligned}
& \operatorname{Pr}\left(F F^{i} \leq t\right)=\operatorname{Pr}\left(\hat{F}^{i}\left(m^{i}\right) \leq t\right) . \\
& \text { If } \hat{F}^{i}=F^{i} \text { : } \\
& \operatorname{Pr}\left(F F^{i} \leq t\right)=\operatorname{Pr}\left(m^{i} \leq\left\{F^{i}\right\}^{-1}(t)\right) \\
& =\operatorname{Pr}\left(D_{k}^{i} \leq\left\{F^{i}\right\}^{-1}(t) \forall k=1, \ldots, N^{i}\right) \\
& =\left[F\left(\left\{F^{i}\right\}^{-1}(t)\right)\right]^{N^{i}} \\
& =t^{N^{i}}
\end{aligned}
$$


Acknowledgements. The authors would to acknowledge MétéoFrance, Météo-Swiss and Instituto Nacional de Meteorologa for the daily data sets. The referees are thanked for their helpful comments. F. Garavaglia would like to warmly acknowledge A. Mantovan for Fig. 4 and T. Mathevet for philosophical and hydrological discussions on the proposed criteria.

Edited by: R. Merz

\section{References}

Akaike, H.: A new look at the statistical model identification. IEEE Transactions on Automatic Control, 19, 6, 1974.

Alexandersson, H.: A homogeneity test applied to precipitation data, J. Climatol., 6, 661-675, 1986.

Anderson, T. W. and Darling, D. A.: Asymptotic theory of certain goodness-of-fit criteria based on stochastic processes, Annals of Mathematical Statistics., 23, 193-212, 1952.

Bardossy, A., Duckstein, L., and Bogardi, I.: Fuzzy rule-based classification of atmospheric circulation patterns. International Journal of Climatology., 15, 1087-1097, 1995.

Bartholmes, J. C., Thielen, J., Ramos, M. H., and Gentilini, S.: The european flood alert system EFAS - Part 2: Statistical skill assessment of probabilistic and deterministic operational forecasts, Hydrol. Earth Syst. Sci., 13, 141-153, doi:10.5194/hess-13-1412009, 2009.

Boé, J. and Terray, L.: A weather type approach to analysing winter precipitation in France: twentieth century trends and influence of anthropogenic forcing, J. Climate, 21, 3118-3133, 2008.

Bois, P.: Contrôle de séries chronologiques corrélées par étude du cumul des résidus de la corrélation, II Journées Hydrologiques de l'ORSTOM, 89-1000, 1976.

Boughton, W. and Droop, O.: Continuous simulation for design flood estimation-a review. Environmental Modelling \& Software., 18, 309-318, 2003.

CFGB: Design flood determination by the gradex method. 18th congress CIGB-ICOLD n2, nov., Bulletin du Comité Français des Grands Barrages-FRCOLD News, 96, 1994.

Coles, S.: An introduction to statistical modeling of extreme values. Springer, London, 2001.

Coles, S., Perricchi, L., and Sisson, S.: A fully probabilistic approach to extreme rainfall modelling, J. Hydrol., 273, 35-50, 2003.

Cramer, H.: On the composition of elementary errors, Skand. Aktuarietids., 11, 13-74 and 141-180, 1928.

Darling, D.A.: The Kolmogorov-Smirnov, Cramer-von Mises Tests. Annals of Mathematical Statistics, 28, 823-838, 1957.

Di Baldassarre, G., Laio, F., and Montanari, A.: Design flood estimation using model selection criteria, Phys. Chem. Earth, 34, 606-611, 2009.

Djerboua, A. and Lang, M.: Scale parameter of maximal rainfall distribution: comparison of three sampling techniques. Revue des Sciences de l'Eau, 20, 111-125, 2007.

Efron, B.: Bootstrap methods: Another look at the jackknife. The Annals of Statistics?, 7, 1-26, 1979.

Garavaglia, F., Gailhard, J., Paquet, E., Lang, M., Garon, R., and Bernardara, P.: Introducing a rainfall compound distribution model based on weather patterns sub-sampling. Hydrol. Earth
Syst. Sci. Discuss., 7, 313-344, doi:10.5194/hessd-7-313-2010, 2010.

Garçon, R.: Oral communication. Statistical and Bayesian Methods in Hydrological Sciences. A joint UNESCO International Conference in honor of Jacques Bernier, September 11-13, Paris, 1995.

Gelman, A., Carlin, J. B., Stern, H. S., and Rubin, D. B.: Bayesian data analysis. Chapmann \& Hall London, 1995.

Gottardi, F.: Estimation statistique et réanalyse des précipitations en montagne. PhD Thesis. Polytechnic Institute of Grenoble, p. 252, Grenoble, 2009.

Guillot P. and Duband D.: La méthode du gradex pour le calcul de la probabilité des crues à partir des pluie, AISH Red Book, 84, 560, 1967.

Hamed, K.: Exact distribution of the Mann-Kendall trend test statistic for persistent data. J. Hydrol., 365, 86-94, 2009.

Karagiannidis, A., Karacostas, T., Maheras, P., and Makrogiannis, T.: Trends and seasonality of extreme precipitation characteristics related to mid-latitude cyclones in Europe, Adv. Geosci., 20, 39-43, doi:10.5194/adgeo-20-39-2009, 2009.

Kendall, M. G.: Rank correction methods. Griffin, London, 1975.

Khamis, H. J.: The delta-corrected Kolmogorov-Smirnov test for the two-parameter Weibull distribution, J. Appl. Stat., 24, 301301, 1997.

Kolmogorov, A. N.: Confidence limits for an unknown distribution function. Annals of Mathematical Statistics., 12, 461-463, 1941.

Kumaraswamy, P.: A generalized probability density function for double-bounded random processes. Journal of Hydrology. 46, 79-88, 1980.

Laio, F.: Cramer-von Mises and Anderson-Darling goodness of fit tests for extreme value distribution with unknown parameters, Water Resour. Res., 40, W09308, doi:10.1029/2004WR003204, 2004.

Laio F., Di Baldassarre, G., and Montanari, A.: Model selection techniques for the frequency analysis of hydrological extremes. Water Resour. Res., 45, W07416, ISSN:0043-1397, doi:10.1029/2007WR006666, 2009.

Lang, M. and Desurosne, I.: Esquisse des risques de crues a l'échelle euro-méditerranéenne: les premiers résultats du programme FRIEND-AHMY exploitant les modèles AGREGEE et TPG. 23emes Journées de l'hydrauliques, Congrès SHF Crues et Inondations, Nimes 14-15-16 September, 1994.

Lang, M., Ouarda, T. B. M. J., and Bobée, B.: Towards operational guidelines for over-threshold modeling. J. Hydrol., 225, 103-117, 1999.

Lang M., Renard, B., Sauquet, E., Bois, P., Dupeyrat, A., Laurent, C., Mestre, O., Niel, H., Neppel, L., and Gailhard J.,: A national study on trends and variations of French floods and droughts, IAHS Publication, 308, 514-519, 2006.

Lehmann, E. L.: Nonparametrics, Statistical Methods Based on Racks. Holden-Day, Inc, California, 1975.

Leonard, M.; Metcalfe, A., and Lambert, M.: Frequency analysis of rainfall and streamflow extremes accounting for seasonal and climatic partitions, J. Hydrol., 348, 135-147, 2008.

Liao, M. and Shimokawa, T.: A new goodness-of-fit test for type-1 extreme-value and 2-parameter Weibull distributions with estimated parameters, J. Stat. Comput. Sim., 64, 23-48, 1999.

Lilliefors, H.: On the Kolmogorov-Smirnov test for normality with mean and variance unknown, J. Am. Stat. Assoc., 62, 399-402, 
1967.

Linderson, M.: Objective classification of atmospheric circulation over southern Scandinavia, Int. J. Climatol., 21, 155-169, 2001.

Littmann, T.: An empirical classification of weather types in the Mediterranean Bassin and their interrelation with rainfall, Theor. Appl. Climatol., 66, 161-171, 2000.

Madsen, H., Rosbjerg, D., and Harremoës, P.: Application of the Bayesian approach in regional analysis of extreme rainfalls, Stochastic Environmental Research and Risk Assessment., 9, 77-88, 1995.

Mann, H. B.: Nonparametric tests against trend, Econometrica, 13, 245-259, 1945.

Martinez, C., Campins, J., Jansà, A., and Genovés, A.: Heavy rain events in the Western Mediterranean: an atmospheric pattern classification, Adv. Sci. Res., 2, 61-64, 2008.

Martins, E. A. and Stendinger, J. R.: Generalized maximum likelihood Pareto-Poisson flood risk analysis for partial duration series, Water Resour. Res., 37, 2559-2567, 2001.

Müller, M., Kašspar, M., and Matschullat, J.: Heavy rains and extreme rainfall-runoff events in Central Europe from 1951 to 2002, Nat. Hazards Earth Syst. Sci., 9, 441-450, doi:10.5194/nhess-9-441-2009, 2009.

Nacházel, K.: Estimation Theory in Hydrology and Water Systems (Developments in Water Science), Elsevier Science, 1993.

Paquet, E., Gailhard, J., and Garçon, R.: Evolution de la méthode du GRADEX: approche par type de temps et modélisation hydrologique, La Houille Blanche., 5, 80-90, 2006.

Plackett, R. L.: Karl Pearson and the Chi-Squared Test, Int. Stat. Rev., 51, 59-72, 1983.

Peterson, T. and Easterling, D. R.: Creation of homogeneous composite climatological reference series, Int. J. Climatol., 14, 671679, 1994.

Pujol, N., Neppel, L., and Sabatier, R.: Regional tests for trend detection in maximum precipitation series in the French Mediterranean region, Journal des Sciences Hydrologiques, 52, 956973, 2008.
Ribatet, M., Sauquet, E., Gresillon, J., and Ouarda, T. B. M. J.: Usefulness of the Reversible Jump Markov Chain Monte Carlo Model in Regional Flood Frequency Analysis, Water Resour. Res., 43, W08403, doi:10.1029/2006WR005525, 2007.

Rosbjerg, D. and Madsen, H.: Advanced approaches in PDS/POT modelling of extreme hydrological events in Hydrology: Science \& Practice for the 21th Century., 217-221, British Hydrological Society, London, 2004.

Schwarz, G.: Estimating the dimension of a model, Ann. Stat., 6, 461-464, doi:10.1214/aos/1176344136, 1978.

Shapiro, S. and Wilk, M. B.: An analysis of variance test for normality (complete samples), Biometrika, 52, 3 and 4, 591-611, 1965.

Smirnov, N. V.: Approximate laws of distribution of random variables from empirical data. Uspehi Matem. Nauk., 10, 179-206, 1944.

Sneyers, R.: On the statistical analysis of series of observations. World Meteorological Organisation. Technical note 143, WMO 415, 1990.

Trigo, R. M. and DaCamara, C. C.: Circulation weather types and their influence on the precipitation regime in Portugal, International Journal of Climatology, 20, 1559-1581, 2000.

Wilks, D. S.: Statistical Methods in the Atmospheric Sciences: An Introduction. Academic Press, 467 pp., 1995.

Yarnal, B., Comrie, A. C., Frakes, B., and Brown, D. P.: Developments and prospects in synoptic climatology. Int. J. Climatol. 21, 1923-1950, 2001.

Yue, S., Pilon, P., and Cavadias, G.: Power of the Mann-Kendall and Spearman's rho tests for detecting monotonic trends in hydrological series. J. Hydrol., 259, 254-271, 2002.

Zveryaev, I.: Seasonality in precipitation variability over Europe, J. Geophys. Res., 109(16), d05103, doi:10.1029/2003JD003668, 2004. 